\title{
Hodgkin's lymphoma associated T-cells exhibit a transcription factor profile consistent with distinct lymphoid compartments
}

\author{
Çiğdem Atayar, Anke van den Berg, Tjasso Blokzijl, Marcel Boot, Randy D Gascoyne, Lydia Visser, \\ Sibrand Poppema
}

See end of article for authors' affiliations

Correspondence to:

Professor Sibrand Poppema, Department of Pathology \& Laboratory Medicine,

University Medical Centre Groningen, Hanzeplein 1, PO Box 30.001, 9700 RB

Groningen The Netherlands; s.poppema@rvb.umcg.nl

Accepted 28 November 2006

Published Online First

8 December 2006
Background: Hodgkin's lymphoma $(\mathrm{HL})$ is characterised by an ineffective immune response that is predominantly mediated by $\mathrm{CD} 4^{+} \mathrm{T}$-cells.

Aims: To analyse the expression of the key regulatory T-cell transcription factors (TFs) in the T-cells of HL involved tissues in order to assess the nature of the $T_{H}$ immune response in $\mathrm{HL}$.

Methods and results: By immunohistochemistry, GATA3 was strongly and T-bet exclusively expressed in a subset of interfollicular lymphocytes in the reactive lymphoid tissues. In classical HL (CHL), which is generally located in the interfollicular zones, a predominance of T-bet ${ }^{+}$T-cells and lesser amounts of GATA ${ }^{+}$and cMaf $^{+} \mathrm{T}$-cells was found, concordant with the pattern of the normal interfollicular compartment. In reactive lymphoid tissues, c-Maf was observed mostly in T-lymphocytes within the germinal centres (GCs). Nodular lymphocyte predominance type of Hodgkin's lymphoma (NLPHL) and progressively transformed germinal centres cases, showed a majority of c-Maf ${ }^{+}{ }^{T}$-cells, consistent with the pattern in normal GCs. NLPHL cases uniformly showed c-Maf ${ }^{+} / \mathrm{CD} 57^{+} \mathrm{T}$-cell rosettes around the neoplastic cells; these rosettes were absent in "paragranuloma-type" T-cell/histiocyte rich B-cell lymphoma.

Conclusions: T-cell TF expression profiles of the reactive T-cells in both subtypes of HL are in accordance with the expression profile observed in the distinct lymphoid compartments.
$\mathrm{H}$ odgkin's lymphoma (HL) is categorised into two distinct clinicopathological entities: classical HL (CHL) and nodular lymphocyte predominance type of HL (NLPHL). ${ }^{1}$ Both types of HL are characterised by a minority of neoplastic cells and a vast majority of reactive infiltrating cells. However, these two HL entities differ in features like immunophenotype and genetic characteristics of the Hodgkin and Reed-Sternberg (HRS) cells, distribution of these cells within the involved lymph node, and composition of the reactive cell population. The neoplastic cells of NLPHL, the lymphocytic and/or histiocytic (L\&H) cells, generally reside within follicular structures containing follicular dendritic cells, T-cells and B-cells, whereas the neoplastic cells of CHL, the HRS cells, are predominantly found in the interfollicular zone. The reasons for the confinement of the tumour cells in distinct lymphoid compartments are not known.

The reactive background cells in HL tissues are mostly CD4 positive T-helper $\left(\mathrm{T}_{\mathrm{H}}\right)$ cells. $^{2} \mathrm{HL}$ associated $\mathrm{T}_{\mathrm{H}}$ cells may represent an influx of $\mathrm{T}_{\mathrm{H}}$ cells from the circulation into the lymphoid tissues. In both types of $\mathrm{HL}$, the $\mathrm{T}$-cells rosetting around the neoplastic cells have a helper/inducer memory phenotype $\left(\mathrm{CD}^{+} / \mathrm{CD} 4 \mathrm{RO}^{+}\right)$, express $\mathrm{CD} 28$ and display an activated phenotype with expression of CD69. ${ }^{3}$ Recently, the presence of $\mathrm{CD}^{+} / \mathrm{CD} 25^{+}$and $\mathrm{FOXP}^{+}{ }^{+}$T-regulatory cells among the infiltrating lymphocytes of CHL was described. ${ }^{45}$ The rosetting T-cells around the $\mathrm{L} \& \mathrm{H}$ cells co-express $\mathrm{CD} 57^{3}$ and ${ }_{B C L 6}{ }^{6}$ a $\mathrm{T}_{\mathrm{H}}$ cell immunophenotype exclusively present in germinal centre (GC) $\mathrm{T}_{\text {-cells. }}{ }^{78}$ The biological significance of these T-cell rosettes in NLPHL has not been elucidated. $\mathrm{CD} 4^{+} /$ CD $57^{+}$T-cells of NLPHL are not only found as rosette formations around the $\mathrm{L} \& \mathrm{H}$ cells but are also more widely distributed throughout the nodules of NLPHL.

Progressively transformed germinal centres (PTGC) have been shown to be associated with NLPHL. ${ }^{90}$ PTGC are enlarged disrupted GCs with a predominance of small B-lymphocytes and dispersed centroblasts that may somewhat resemble the
L\&H cells cytologically, but only occasionally are surrounded by $\mathrm{CD} 4^{+} / \mathrm{CD} 57^{+}$T-cells. Moreover, PTGC can be observed in $15 \%$ of patients with NLPHL before, during or after NLPHL, while PTGC occur in only $2 \%$ of patients with CHL. ${ }^{11}$

$\mathrm{T}_{\mathrm{H}}$ cell differentiation is the central process defining the nature of developing immune responses. ${ }^{12}$ GATA3, T-bet (also known as TBX21) and c-Maf (also known as MAF) are the key regulator transcription factors (TFs) that dictate the development of $\mathrm{T}_{\mathrm{H}}$ subsets. ${ }^{12}$ Although the mechanisms of action of the abovementioned T-cell TFs have been extensively studied, ${ }^{12}$ there have been very few ${ }^{13}$ or no reports of their pattern of distribution in normal lymphoid tissues as revealed by immunohistological staining. Previously, we investigated expression of T-cell TFs in HL cell lines and tissues involved by HL and found frequent expression of GATA3 and T-bet in the neoplastic cells of HLs, ${ }^{14}$ a finding confirmed by Dorfman et al. ${ }^{15}$ The aim of the present study was to gain further insight into the nature of the $\mathrm{T}_{\mathrm{H}}$ immune response in HL tissues and to investigate the T-cell TF expression profile of the normal lymphoid tissues. We performed an immunohistochemical profiling of the reactive $\mathrm{T}$-cells in $\mathrm{HL}$ and reactive lymphoid tissues for the three aforementioned T-cell TFs. We extended our analysis by including PTGC cases to investigate the potential relation between PTGC and NLPHL and cases of "paragranuloma type" T-cell/histiocyte rich B-cell lymphoma (T/HRBCL) that morphologically resemble NLPHL. ${ }^{16}$ The expression pattern was evaluated by considering frequency, staining intensity and localisation of positive cells with respect to lymph node architecture and tumour cells.

Abbreviations: $\mathrm{CHL}$, classical Hodgkin's lymphoma; GC, germinal centre; $\mathrm{HL}$, Hodgkin's lymphoma; HRBCL, histiocyte rich B-cell lymphoma; HRS, Hodgkin and Reed-Sternberg; L\&H, lymphocytic and/or histiocytic; NLPHL, nodular lymphocyte predominance type of Hodgkin's lymphoma; PTGC, progressively transformed germinal centre; TF, transcription factor; $\mathrm{T}_{\mathrm{H}}$, $\mathrm{T}$-helper 
Table 1 Antibodies used in the present study

\begin{tabular}{llll}
\hline Transcription factor & Antibody & Source & Dilution \\
\hline T-bet & 4B10 (monoclonal, lgG1) & Prof LH Glimcher, Harvard Medical School, Boston, MA, USA & $1 / 50$ \\
& $52-3567$ (polyclonal) & Zymed Laboratories Inc., San Francisco, CA, USA & $1 / 50$ \\
GATA3 & HG3-31 (monoclonal, lgG1) & Santa Cruz Biotechnology, Santa Cruz, CA, USA & $1 / 50$ \\
c-Maf & SC- 7866 (polyclonal) & Santa Cruz Biotechnology, Santa Cruz, CA, USA & $1 / 50$ \\
CD3 & PS1 (monoclonal, IgG2a) & Ventana Medical Systems Inc., Oro Valley, AZ, USA & prediluted \\
CD57 & NK1 (monoclonal, lgM) & Monosan, Uden, The Netherlands & $1 / 40$ \\
\hline
\end{tabular}

\section{MATERIALS AND METHODS}

\section{Patients and tissue samples}

Formalin-fixed, paraffin-embedded and frozen samples of a series of previously diagnosed HL tissues, comprising 16 CHL ( 7 female, 9 male; age 10-94 years, mean 40 years, median 33 years), 7 NLPHL ( 2 female, 5 male; age 17-73 years, mean 36 years, median 34 years), and 4 PTGC ( 2 female, 2 male; age 2454 years, mean 33 years, median 26 years) cases were obtained from the files of the Department of Pathology \& Laboratory Medicine, University Medical Centre Groningen, The Netherlands, according to institutional guidelines. The CHL group comprised 10 cases of nodular sclerosis type, 3 cases of mixed cellularity type, 1 case of lymphocyte-depleted type, and 2 cases of unclassifiable HL. All NLPHL cases showed a predominant nodular pattern together with minor diffuse areas involving usually less than $10-20 \%$ of the involved lymph node area. Two cases of PTGC had a HL history and 2 cases were de novo cases. In the PTGC cases, $10-25 \%$ of the follicles per tissue section were involved. We also studied 6 cases of "paragranuloma type" T/ HRBCL that were previously described by Rudiger et al. ${ }^{16}$ Tissue blocks of 3 reactive tonsils, 5 reactive lymph nodes and 3 normal adult thymuses were used as control tissues. All samples were anonymised; full ethical approval in accordance with local policy was obtained by the institution's review board for human subject research for obtaining and using these tissue samples.

\section{Immunohistochemistry}

Paraffin-embedded tissue sections ( $4 \mu \mathrm{m}$ thick) were cut, mounted on aminopropyltriethoxysilane-coated slides (LoLaboroptik GmbH, Friedrichsdorf, Germany), dried, deparaffinised in xylene and rehydrated in graded alcohols. Antigen retrieval was carried out in a microwave oven (Amana RS591SS, Salm \& Kipp, Breukelen, The Netherlands) (three times for $15 \mathrm{~min}$ at 300W) in $1 \mathrm{mmol} / \mathrm{l}$ Tris (Merck, Darmstadt, Germany)-EDTA (Serva, Heidelberg, Germany) buffer ( $\mathrm{pH}$ 8.0) for c-Maf and in $10 \mathrm{mmol} / \mathrm{l}$ citrate (Merck) buffer ( $\mathrm{pH} \mathrm{6.0)} \mathrm{for}$ T-bet. After antigen retrieval the slides were incubated for 1 hour at room temperature with the primary antibodies (table 1), followed by $30 \mathrm{~min}$ incubation with the secondary antibody and for an additional $30 \mathrm{~min}$ with the tertiary antibody.

All antibody dilutions were prepared with phosphate buffered saline, pH 7.4 containing 1\% bovine serum albumin. The second step for c-Maf and T-bet was performed with horseradish peroxidase (HRP) labelled goat-anti-rabbit Ig (DAKO, Copenhagen, Denmark) and the third step by HRP labelled rabbit anti-goat Ig (DAKO). Antibody binding was detected by 3,3'-diaminobenzidine (Sigma-Aldrich, Saint Louis, MO, USA) staining. For GATA3 staining $4 \mu \mathrm{m}$-thick sections from frozen reactive tonsils and lymph nodes were airdried, fixed in acetone, and incubated for 1 hour with primary antibody, followed by application of HRP labelled rabbit-antimouse Ig and goat-anti-rabbit Ig (DAKO) incubation steps for 30 min each. 3-Amino-9-ethylcarbazole (Sigma-Aldrich) was used to visualise the positive cells. Positive control tissues as well as negative controls, sections stained in parallel without primary antibody, were used in all instances. The pattern and intensity $(+$, weak; ++ , moderate; or +++ , strong) of the staining was also recorded. PTGC findings reported are the findings observed in the transformed follicles of PTGC cases. Reactive follicles and paracortical areas surrounding the transformed follicles had the same staining pattern as reactive/normal lymphoid tissues.

\section{Double immunostaining}

Double immunostaining was carried out on paraffin sections of reactive tonsils to confirm that $\mathrm{T}$-bet and c-Maf was expressed by T-cells. To detect T-bet and c-Maf, the above mentioned protocol was followed; subsequently the same protocol was repeated using CD3 and CD57 (table 1) which was developed with rabbit-anti-mouse Ig and goat-anti-rabbit Ig conjugated to alkaline phosphatase (DAKO) and Fast Blue. No counter stain was used. Single staining for T-bet, c-Maf, CD3, and CD57 was also performed by applying all the other steps used for the double staining to exclude cross-reaction of the different antibodies. Negative control staining (incubation without primary antibody) was performed routinely.

\section{RESULTS}

\section{Strong expression of $\mathrm{T}$-bet in reactive infiltrate of $\mathrm{CHL}$} cases

In thymic cortex and medulla only a few cells exhibited moderate to strong T-bet positivity (fig lA, table 2).

In GCs of tonsil or reactive lymph node, virtually no T-bet staining was observed (fig 1B). Staining was restricted to the interfollicular compartments as well as the subepithelial area in the tonsil. In reactive lymph nodes, interfollicular lymphocytes as well as marginal sinus lymphocytes stained intensely for Tbet and were especially prominent in vascular and perivascular areas. In addition to the mature lymphocytes, some macrophages also showed reactivity with T-bet antibody in moderate intensity. In PTGC cases strongly stained cells were present in the interfollicular area similar to reactive lymph nodes. Additionally, a few scattered moderately or strongly stained T-bet ${ }^{+}$cells were observed in broken up GCs as well as in the enlarged mantle zone (fig $1 \mathrm{C}$ ).

NLPHL cases showed weak (fig 1D) to moderate staining in $5-10 \%$ of the non-neoplastic lymphocytes which were preferentially located in perivascular areas. One case in which we observed strong T-bet immunoreactivity in $25-50 \%$ of $\mathrm{L} \& \mathrm{H}$ cells $^{14}$ also exhibited intense $\mathrm{T}$-bet reactivity in reactive $\mathrm{T}$ lymphocytes. In $14 \mathrm{CHL}$ cases the majority of T-lymphocytes showed moderate to strong (fig lE) T-bet reactivity (table 3 ).

In two cases T-bet expression was observed in less than $10 \%$ of the T-lymphocytes. Remarkably, the HRS cells of these two cases were also negative for T-bet staining, whereas in the rest of the cases HRS cells also expressed T-bet. Double immunostaining with $\mathrm{CD} 3$ on tonsil sections revealed that $\sim 20 \%$ of the $\mathrm{CD}^{+}{ }^{+} \mathrm{T}$-cells were $\mathrm{T}$-bet ${ }^{+}$. These $\mathrm{T}$-bet ${ }^{+}$cells were exclusively located in the interfollicular area. On the other hand, $\sim 50 \%$ of the T-bet ${ }^{+}$cells were expressing CD3 in tonsil. On CHL sections 

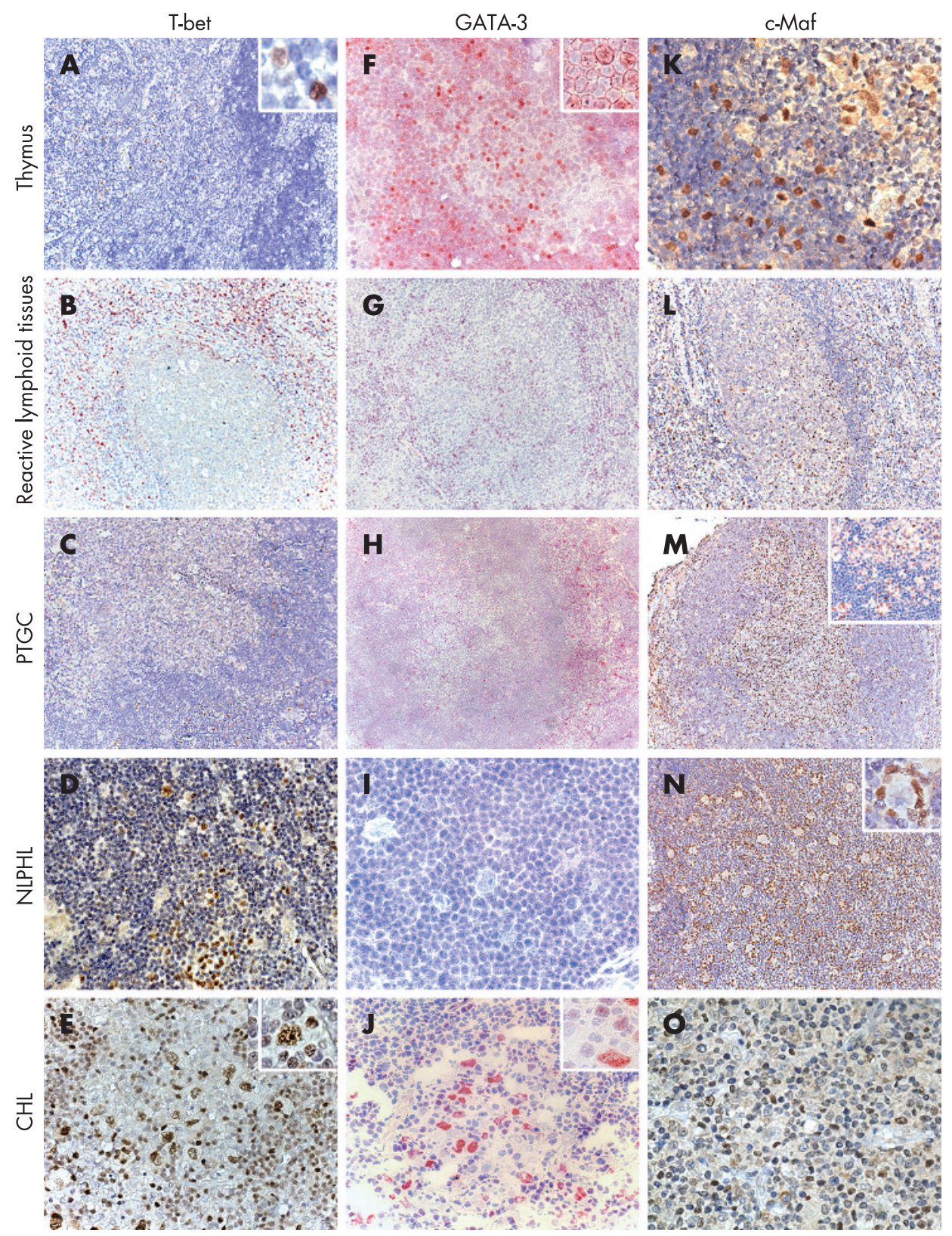

Figure 1 Immunohistochemical detection of T-cell TFs in thymus $(A, F, K)$, reactive lymphoid tissues $(B, G, L)$, progressively transformed germinal centre (PTGC; C, H, M), nodular lymphocyte predominance type of Hodgkin's lymphoma (NLPHL; D, I, N), and classical Hodgkin's lymphoma (CHL; E, J, O). T-bet immunostaining (A-E), GATA3 immunostaining (F-J), c-Maf immunostaining (K-O). Original magnification: $\times 25$ for $\mathrm{H}, \mathrm{M} ; \times 50$ for $A-\mathrm{C}, \mathrm{G}, \mathrm{N} ; \times 100$ for $E, F, J, K ; \times 157.5$ for D, I, O; $\times 100$ for inset $M, \times 157.5$ for all other insets. For GATA3 frozen sections, for c-Maf and T-bet staining paraffin sections were used. Only a few cells showed T-bet immunoreactivity in thymus (A). In reactive lymphoid tissues T-bet positivity was restricted to the interfollicular compartment (B). Although in transformed follicles of PTGC and NLPHL background cells T-bet expression was not remarkable (C, D), in CHL reactive lymphocytes T-bet was the most predominantly expressed T-cell TF (E). GATA3 was expressed abundantly in thymus (F) and in various compartments of reactive lymphoid tissues (G); however in both subtypes of HL $(I, J)$ (remarkably in NLPHL (I)), low numbers of GATA3 ${ }^{+}$T-cells were detected. Scattered c$\mathrm{Maf}^{+}$cells were observed in thymus cortex and medulla (K). In reactive lymphoid tissues c-Maf staining was prominently observed in the light zone and beneath the mantle zone (L). In PTGC and NLPHL cases, numbers of c-Maf ${ }^{+}$cells were remarkably increased (M, N). Few c-Maf ${ }^{+}$rosettes were observed in borders of transformed follicles of PTGC (M inset), whereas in NLPHL cases they were observed extensively in all cases (N inset). c-Maft cells in CHL cases were fewer in number than NLPHL cases and no obvious rosetting pattern was observed in positive cells (O).

(fig 2A), $80-90 \%$ of the $\mathrm{CD}^{+}$cells were also $\mathrm{T}^{- \text {bet }^{+}}$and more than $90 \%$ of the T-bet ${ }^{+}$cells were $\mathrm{CD}^{+}$.

Low levels of GATA3 expression in T-cell infiltrates of $\mathrm{HL}$ In thymus most cortical thymocytes had cytoplasmic GATA3 and only some had nuclear GATA3, while medullary lymphocytes had exclusively nuclear GATA3 staining (fig $1 F$ ). The staining in thymus was generally moderate to strong in intensity. In tonsil and reactive lymph nodes strong nuclear GATA3 immunoreactivity was seen in scattered extrafollicular lymphocytes (table 2). Eosinophils, with strong cytoplasmic GATA3 staining, were also detected in interfollicular areas. In the light and dark zones of GCs a staining of weak to moderate intensity was observed, especially in larger lymphocytes. In the mantle zone some of the lymphocytes showed weak GATA3 immunoreactivity (fig $1 G$ ). In PTGC both transformed follicles and enlarged mantle zone displayed moderately or weakly interspersed positive cells, whereas in interfollicular areas strongly GATA3 ${ }^{+}$lymphocytes were observed similar to the reactive lymph node (fig $\mathrm{lH}$ ). 
Table 2 Immunohistochemical expression pattern of T-cell transcription factors (TFs) in thymus, reactive lymphoid tissues and progressively transformed germinal centres (PTGC)

\begin{tabular}{|c|c|c|c|c|c|c|}
\hline \multirow[b]{2}{*}{ Tissue } & \multicolumn{2}{|l|}{ T-bet } & \multicolumn{2}{|l|}{ GATA3 } & \multicolumn{2}{|l|}{ c-Maf } \\
\hline & $\begin{array}{l}\% \text { Positive } \\
\text { cells }\end{array}$ & Intensity & $\begin{array}{l}\% \text { Positive } \\
\text { cells }\end{array}$ & Intensity & $\begin{array}{l}\% \text { Positive } \\
\text { cells }\end{array}$ & Intensity \\
\hline \multicolumn{7}{|l|}{ Thymus } \\
\hline Cortex & $1-2$ & ++ & $50-75$ & ++ & $1-5$ & +++ \\
\hline Medulla & $1-3$ & ++ & $10-25$ & ++ & $5-10$ & ++ \\
\hline \multicolumn{7}{|l|}{ Reactive lymphoid tissue } \\
\hline GCs & - & - & $1-5$ & ++ & $5-10$ & $+++\dagger$ \\
\hline Mantle zone & $<1$ & ++ & $1-5$ & + & $1-2$ & + \\
\hline Extrafollicular compartment & $3-5$ & $++\ddagger$ & $5-10$ & ++ & $3-5$ & +++ \\
\hline \multicolumn{7}{|l|}{ PTGC* } \\
\hline Transformed GCs & $<1$ & ++ & $1-5$ & ++ & $10-25$ & +++ \\
\hline Expanded mantle zone & $1-2$ & +++ & $1-5$ & ++ & $2-3$ & $++\S$ \\
\hline Extrafollicular compartment & $3-5$ & ++ & $5-10$ & ++ & $3-5$ & ++ \\
\hline \multicolumn{7}{|c|}{$\begin{array}{l}\text { GC, germinal centre. } \\
- \text {, negative; }+ \text {, weakly positive; }++ \text {, moderately positive; }+++ \text {, strongly positive. } \\
\text { *PTGC findings denote the findings observed in transformed follicles of PTGC cases. Reactive follicles and paracortical } \\
\text { areas surrounding the transformed follicles had the same staining pattern as reactive/normal lymphoid tissues. } \\
\text { tc-Maf expression in GC was detected mainly in light zone of GCs. Some of the GC macrophages showed moderate } \\
\text { expression of c-Maf. } \\
\text { †Strongly T-bet }{ }^{+} \text {cells were noted, especially in perivascular areas of sinusoids and marginal sinus of the reactive/normal } \\
\text { lymph nodes. } \\
\S c-M^{+} \text {cells were observed as collarettes or single cells. }\end{array}$} \\
\hline
\end{tabular}

GATA3 immunostaining was observed at very low numbers of NLPHL associated T-cells (fig II). The positive T-lymphocytes within the nodules were distributed randomly with no spatial relation to tumour cells. In most CHL cases $10-25 \%$ of background lymphocytes were $\mathrm{GATA}^{+}$(table 3 ). In two cases, $25-50 \%$ of the reactive infiltrate showed a moderate staining for GATA3. The positive T-lymphocytes appeared to be distributed randomly with no specific relation to HRS cells (fig $1 \mathrm{~J}$ ).

\section{Striking expression of c-Maf in NLPHL and PTGC, but not in "paragranuloma type" $\mathrm{T} / \mathrm{HRBCL}$}

In thymus, scattered larger thymocytes in the cortex stained strongly with c-Maf, whereas in the medulla large cells as well as interspersed smaller cells were stained with weak to moderate intensity (fig $1 \mathrm{~K}$, table 2 ). ${\mathrm{c}-M a f^{+}}^{+} \mathrm{T}$-cells were predominantly detected in the light zone of GCs and just beneath the follicular mantle, even though they were also observed in the other compartments including the mantle zone and interfollicular area (fig lL). Some of the GC macrophages were also stained in moderate intensity. When we performed double immunostaining on tonsil sections with CD3, 90-95\% of

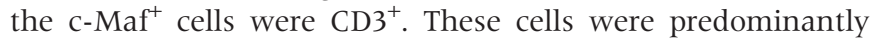
located in GCs. Moreover, $5-10 \%$ of the $\mathrm{CD}^{+}{ }^{+} \mathrm{T}$-cells were c-Maf co-positive. Double immunostaining with CD57 on tonsil sections revealed that $60-70 \%$ of the $\mathrm{c}-\mathrm{Maf}^{+}$cells were coexpressing CD57 and these cells were exclusively located within the GCs. In total more than $90 \%$ of the CD $57^{+}$T-cells were cMaf co-positive. In the interfollicular areas, intensely stained cells were interspersed. Strongly c-Maf ${ }^{+}$cells were highly increased in numbers in transformed follicles of PTGC cases (fig 1M). In contrast to normal GC, there was no regional distribution of $\mathrm{c}-\mathrm{Maf}^{+}$cells in PTGC. Although few in number, some $\mathrm{c}-\mathrm{Maf}^{+}$collarettes with strong staining intensity were observed around large lymphocytes in PTGC (fig $1 \mathrm{M}$, inset). These scattered rosette like formations were found especially in the enlarged mantle zone. Single cells that were c-Maf ${ }^{+}$were also observed in the expanded mantle zone.

Table 3 Immunohistochemical expression of T-cell transcription factors (TFs) in reactive lymphoid cells in tissues involved by Hodgkin's lymphoma (HL)

\begin{tabular}{|c|c|c|c|c|c|c|c|}
\hline \multirow[b]{2}{*}{ HL subtype } & \multirow{2}{*}{$\begin{array}{l}\% \text { Positive } \\
\text { cells }\end{array}$} & \multicolumn{2}{|l|}{ T-bet } & \multicolumn{2}{|l|}{ GATA3 } & \multicolumn{2}{|l|}{ c-Maf } \\
\hline & & Cases/total & Intensity & Cases/total & Intensity & Cases/total & Intensity \\
\hline $\mathrm{NLPHL}^{*}$ & $\begin{array}{l}<10 \\
10-25 \\
25-50 \\
50-75 \\
>75\end{array}$ & $\begin{array}{l}5 / 6 \\
1 / 6\end{array}$ & $\begin{array}{l}+ \\
+++\end{array}$ & $7 / 7$ & + & $\begin{array}{l}3 / 6 \\
2 / 6 \\
1 / 6\end{array}$ & $\begin{array}{l}++ \\
+++ \\
+++\end{array}$ \\
\hline $\mathrm{CHL} †$ & $\begin{array}{l}<10 \\
10-25 \\
25-50 \\
50-75 \\
>75\end{array}$ & $\begin{array}{l}2 / 16 \\
3 / 16 \\
7 / 16 \\
4 / 16\end{array}$ & $\begin{array}{l}+ \\
++ \\
++ \\
++\end{array}$ & $\begin{array}{l}5 / 12 \\
5 / 12 \\
2 / 12\end{array}$ & $\begin{array}{l}+ \\
++ \\
++\end{array}$ & $\begin{array}{l}1 / 13 \\
8 / 13 \\
3 / 13 \\
1 / 13\end{array}$ & $\begin{array}{l}+ \\
+ \\
++ \\
++\end{array}$ \\
\hline
\end{tabular}

NLPHL, nodular lymphocyte predominance type of Hodgkin's lymphoma; CHL, classical Hodgkin's lymphoma. + , weakly positive; ++ , moderately positive; +++ , strongly positive.

Percentages refer to the percentage of immunoreactive cells relative to all infiltrating cells.

*Most rosetting cells in NLPHL cases were moderately or strongly c-Maf'.

†There was no clear difference among different subsets of $\mathrm{CHL}$ according to number of positive cells, distribution or intensity of T-cell TF expression. 

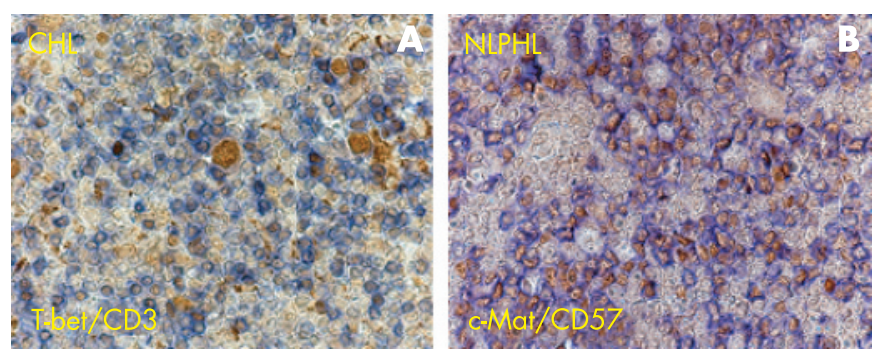

Figure 2 Double immunostaining of Hodgkin's lymphoma sections with Tcell markers. (A) Double staining for T-bet and CD3 in classical Hodgkin's lymphoma (CHL) showed that the vast majority of the T-bet expressing background cells of $\mathrm{CHL}$ were $\mathrm{CD} 3^{+} \mathrm{T}$-cells. Hodgkin and Reed-Sternberg cells were also strongly T-bet ${ }^{+}$. (B) Double staining of nodular lymphocyte predominance type of Hodgkin's lymphoma (NLPHL) with c-Maf and CD57 revealed the abundant presence of $\mathrm{c}-\mathrm{Maf}{ }^{+} / \mathrm{CD} 57^{+}$phenotype T-cells as rosette formations around lymphocytic and/or histiocytic cells, or widely distributed in the NLPHL nodule. Original magnification $\times 157.5$.

In the majority of NLPHL cases, $25-50 \%$ of the lymphocytes showed moderate to strong expression of c-Maf (table 3 ). High numbers of positive cells with a strong staining intensity for cMaf were detected preferentially within the neoplastic follicles and in the lymphocytes, forming rosettes around the L\&H cells (fig $1 \mathrm{~N}$ and inset). A lower number of $\mathrm{c}-\mathrm{Maf}^{+}$cells were observed outside of the nodules of NLPHL. While strongly expressed on rosetting cells, a weak to moderate staining intensity was observed on non-rosetting lymphocytes. Double staining of NLPHL sections with c-Maf and CD57 revealed the abundant presence $\left(90-95 \%\right.$ of the c-Maf ${ }^{+}$cells were CD $57^{+}$and almost all $\mathrm{CD} 57^{+}$cells were c-Maf ${ }^{+}$) of c-Maf ${ }^{+} / \mathrm{CD} 57^{+}$phenotype $\mathrm{T}$-cells as rosette formations around $\mathrm{L} \& \mathrm{H}$ cells or distributed throughout the nodules (fig 2B). Of 13 CHL cases, nine exhibited c-Maf staining in $\leqslant 10-25 \%$ of the T-lymphocytes (fig 10). c- $\mathrm{Maf}^{+}$cells in CHL were distributed randomly throughout the involved lymph node. Scattered c-Maf ${ }^{+} \mathrm{T}$-cells in the vicinity of the HRS cells were also observed, but there were no extensive rosette formations as in NLPHL. Paragranuloma type of T/HRBCL cases revealed a "CHL pattern" of staining with c-Maf antibody although they show histological similarity to NLPHL. In general, about $15-20 \%$ of the T-cells were c-Maf ${ }^{+}$, which were randomly distributed; no c$\mathrm{Maf}^{+}$collarettes were observed in the six cases of paragranuloma type of T/HRBCL (fig 3).

\section{DISCUSSION}

In the present study, we analysed the expression pattern of three T-cell TFs, considered to be important in $\mathrm{T}_{\mathrm{H}}$ lineage commitment, in the reactive cells of HL. T-bet ${ }^{17}$ and GATA3 ${ }^{18} 19$
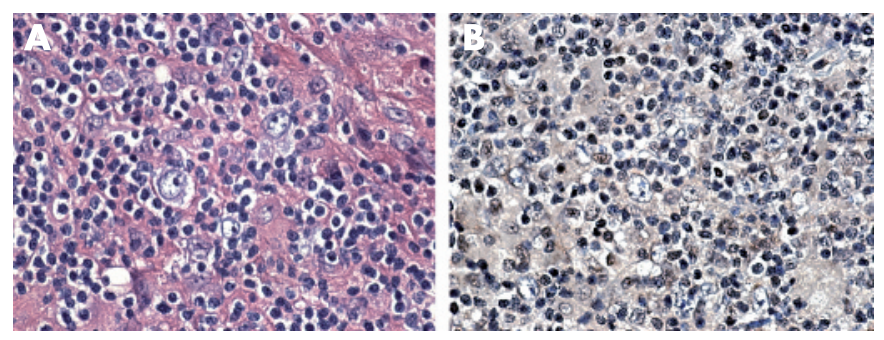

Figure 3 Paragranuloma type T/HRBCL. (A) H\&E staining of "paragranuloma" type T/HRBCL with neoplastic cells resembling "popcorn" cells of NLPHL and the surrounding non-neoplastic

lymphocytes. (B) c-Maf staining of paragranuloma type T/HRBCL showed that the c-Maf ${ }^{+}$cells were less in number (in this case $\sim 20 \%$ ) compared to $\mathrm{NLPHL}$. c-Maf ${ }^{+}$cells were randomly distributed and no c-Maf ${ }^{+}$rosettes were present. Original magnification $\times 157.5$. were identified as master factors regulating induction of $T_{H} 1$ and $\mathrm{T}_{\mathrm{H}} 2$ pathways respectively. c-Maf is a basic leucine zipper transcription factor that is expressed in $\mathrm{T}_{\mathrm{H}} 2$ cells and has a selective function in regulation of interleukin 4 (IL4) transcription. ${ }^{20}$ c-Maf expression has also been reported in multiple myeloma, ${ }^{21}$ and T-bet expression has been found in normal Bcells $^{22}$ and some B-cell neoplasms. ${ }^{13}{ }^{14}$ To show the T-cell nature of the positive cells we performed double immunostainings with T-cell specific markers on control tonsil sections. These revealed that the vast majority of the $\mathrm{c}-\mathrm{Maf}^{+}$and $\mathrm{T}$-bet ${ }^{+}$cells were indeed of T-cell origin.

We have shown that the reactive T-cells in CHL cases are predominantly immunoreactive for T-bet. The T-cells in CHL include cells expressing IL2, IL10, IL13, IFN, TGFB $\beta 1,{ }^{2}$ and FOXP $3^{5}$ and can be regarded as having a $\mathrm{T}_{\mathrm{H}} 2$-like or immunoregulatory phenotype. ${ }^{43}$ This cytokine profile is not consistent with the "master $\mathrm{T}_{\mathrm{H}} \mathrm{l}$ transcription regulator" ${ }^{17}$ - T-bet expression in the reactive T-cells of CHL. However, $T$-bet expression is not necessarily incompatible with $\mathrm{T}_{\mathrm{H}} 2$ cytokine production since retroviral expression of T-bet into $\mathrm{T}_{\mathrm{H}} 2$ cells does not extinguish expression of GATA3 or $\mathrm{T}_{\mathrm{H}} 2$ cytokines. ${ }^{24}$ Moreover, T-bet immunoreactivity in the reactive T-cells of $\mathrm{CHL}$ does not indicate a $\mathrm{T}_{\mathrm{H}} \mathrm{l}$ type immune response per se; recently a new type of $\mathrm{CD}^{+}$/ $\mathrm{CD}_{25} 5^{-}$adaptive T-regulatory cell has been identified that is phenotypically related to $\mathrm{T}_{\mathrm{H}} \mathrm{l}$ cells with expression of $\mathrm{T}$-bet, FOXP3, ICOS, IL10 and IFN $\gamma .{ }^{25}$ Raised levels of FOXP3 ${ }^{+} \mathrm{T}$-cells were indeed described in CHL, albeit in a low percentage of cases. ${ }^{5}$ Therefore, it is possible that the TF expression repertoire of the reactive $\mathrm{T}$-lymphocytes of CHL indicates a specific subpopulation of T-regulatory cells.

In reactive lymphoid tissues, $\mathrm{T}^{- \text {bet }^{+}}$as well as $\mathrm{FOXP}^{+} \mathrm{T}$-cell populations were observed solely in the interfollicular compartment of the reactive lymphoid tissues ${ }^{5}$; this is consistent with the presence of CHL in the interfollicular compartment of the lymph nodes.

In the CHL background population, we also detected GATA3 ${ }^{+}$ and c-Maf ${ }^{+}$cells at low numbers which did not exhibit a specific distribution pattern. The presence of T-cells with a different T-cell TF expression repertoire suggests various subpopulations with different specificities and functions. On the other hand, during the T-cell differentiation process, before achieving restricted expression in the fully polarised cell, persistent co-expression of both types of T-cell TFs has been reported in both $\mathrm{T}_{\mathrm{H}}$ subsets. ${ }^{26}$ Therefore, it is also plausible that part of the reactive T-cells of $\mathrm{HL}$ are not yet fully committed or have retained the ability to activate and express $\mathrm{T}_{\mathrm{H}} 1$ and $\mathrm{T}_{\mathrm{H}} 2$ type TFs together.

We have shown that $\mathrm{c}-\mathrm{Maf}^{+} \mathrm{T}$-cells are predominantly located within the GCs of the reactive lymphoid tissues. L\&H cells of NLPHL have characteristics of GC B-cells, and the nodules of NLPHL are considered altered germinal centres. ${ }^{27}$ Thus, L\&H cells, surrounded by c-Maf ${ }^{+} \mathrm{T}$-cells, proliferate in a GC-like environment. The $\mathrm{CD} 57^{+} \mathrm{T}$-cell rosetting pattern is a distinct and well known feature of NLPHL, ${ }^{3}$ but this is the first demonstration of $\mathrm{c}-\mathrm{Maf}^{+} / \mathrm{CD} 57^{+} \mathrm{T}$-cell rosettes around $\mathrm{L} \& \mathrm{H}$ cells. Carbone et $a^{28}$ showed the presence of MUMl/IRF4 ${ }^{+} \mathrm{T}$ cells, which are located mainly in close proximity to L\&H cells as rosettes. c-Maf is a specific TF for IL4, ${ }^{20}$ and MUMI/IRF4 synergises with NFATc2 and c-Maf, to augment IL4 promoter activity as well as to elicit significant levels of endogenous IL4 production. ${ }^{29}$ However, we have recently reported that in contrast to their tonsillar counterparts, $\mathrm{CD} 4^{+} / \mathrm{CD} 57^{+} \mathrm{T}$-cells of NLPHL do not express IL4 even after PMA-ionomycine activation. $^{2}$ This suggests that in NLPHL, c-Maf and MUMl/ IRF4 induced expression of IL4 is blocked. c-Maf also has a transforming ability, ${ }^{30}$ and has been shown to function as an oncogene. ${ }^{31}$ The functional significance of $\mathrm{c}-\mathrm{Maf}^{+} \mathrm{T}$-cell rosettes in NLPHL cases remains to be elucidated. 
NLPHL can histologically and immunophenotypically resemble a subset of T/HRBCL cases. ${ }^{32}$ To investigate whether the presence of $\mathrm{c}-\mathrm{Maf}^{+}$rosettes is of value in the differential diagnosis between T/HRBCL and NLPHL, we have stained a specific subtype of cases referred to as "paragranuloma type" of T/HRBCL $L^{16}$ for c-Maf antibody. None of the six paragranuloma type of T/HRBCL cases showed c-Maf ${ }^{+}$rosettes but rather showed a "CHL pattern" of cMaf staining. Therefore, the presence of $\mathrm{c}-\mathrm{Maf}^{+}$rosettes may help to distinguish NLPHL from paragranuloma type of T/HRBCL. In fact, three immunophenotypic features can be used to distinguish NLPHL from T/HRBCL, including two markers of the neoplastic B cells (LSP-1, PU.1) (3) $^{33}$ and one differentially expressed by the nonneoplastic T cells (c-Maf).

PTGC have been consistently been associated with NLPHL. ${ }^{10}$ Our results further support the hypothesis that PTGC and NLPHL may both be manifestations of an abnormal follicular centre reaction. c-Maf staining was most pronounced in the light zone of GC of the reactive lymph nodes (tables 2 and 3). In transformed follicles of PTGC cases there was an increased frequency of c-Maf ${ }^{+}$ T-cells together with loss of zonal distribution of c-Maf reactivity. Frequent $\mathrm{C}-\mathrm{Maf}^{+} \mathrm{T}$-cell rosettes around $\mathrm{L} \& \mathrm{H}$ cells was a common characteristic of all NLPHL cases, and c-Maf ${ }^{+}$rosette like formations were also detected between broken-up borders of GC and the mantle zone in transformed follicles of PTGC, albeit at low frequencies. PTGC and NLPHL nodules contained very few GATA3 $^{+}$and T-bet ${ }^{+}$T-lymphocytes, which is consistent with an enlarged mantle zone like B-cell rich area.

In conclusion, we have shown that CHL associated lymphocytes are mostly T-bet expressing T-cells, whereas NLPHL cases have a high frequency of $\mathrm{c}-\mathrm{Maf}^{+} / \mathrm{CD} 57^{+} \mathrm{T}$-cells as rosettes or single cells. The T-cell TF expression pattern of the HL associated T-cells is consistent with the T-cell TF pattern observed in the distinct lymphoid compartments in which HL subtypes are diagnosed. This implies that background T-cells of HL are not randomly distributed T-cells, but an expansion of the native T-cells or an influx of T-cells with the same profile as the native T-cells in the corresponding lymphoid compartments.

\section{Take-home messages}

- Background T-cells of Hodgkin's lymphoma (HL) are not randomly distributed $\mathrm{T}$-cells: the reactive $\mathrm{T}$-cells in classical Hodgkin's lymphoma (CHL) are predominantly immunoreactive for T-bet and in NLPHL for c-Maf.

- T-cell transcription factor profiles of $\mathrm{HL}$ indicate that the two types of $\mathrm{HL}$ are two different diseases within two distinctive lymphoid compartments.

- Raised numbers of $\mathrm{c}-\mathrm{Maf}^{+} / \mathrm{CD} 57^{+} \mathrm{T}$-cell rosettes are characteristic for NLPHL.

\section{Authors' affiliations}

Çiğdem Atayar, Anke van den Berg, Tjasso Blokzijl, Marcel Boot, Lydia Visser, Sibrand Poppema, Department of Pathology \& Laboratory Medicine, University of Groningen and University Medical Centre Groningen, The Netherlands

Randy D Gascoyne, Department of Pathology, University of British Columbia and British Columbia Cancer Agency, Vancouver, BC, Canada

Funding: This study was supported by the Groningen University Institute for Drug Exploration.

Competing interests: None.

\section{REFERENCES}

1 Harris NL. Hodgkin's lymphomas: classification, diagnosis, and grading. Semin Hematol 1999;36:220-32.
2 Atayar C, Poppema S, Visser L, et al. Cytokine gene expression profile distinguishes CD4(+)/CD57(+) T cells of the nodular lymphocyte predominance type of Hodgkin's lymphoma from their tonsillar counterparts. J Pathol 2006;208:423-30.

3 Poppema S. The nature of the lymphocytes surrounding Reed-Sternberg cells in nodular lymphocyte predominance and in other types of Hodgkin's disease. Am J Pathol 1989;135:351-7.

4 Marshall NA, Christie LE, Munro LR, et al. Immunosuppressive regulatory T cells are abundant in the reactive lymphocytes of Hodgkin lymphoma. Blood 2004; 103:1755-62.

5 Alvaro T, Lejeune M, Salvado MT, et al. Outcome in Hodgkin's lymphoma can be predicted from the presence of accompanying cytotoxic and regulatory $T$ cells. Clin Cancer Res 2005;11:1467-73.

6 Falini B, Bigerna B, Pasqualucci L, et al. Distinctive expression pattern of the BCL6 protein in nodular lymphocyte predominance Hodgkin's disease. Blood 1996;87:465-71.

7 Bowen MB, Butch AW, Parvin CA, et al. Germinal center T cells are distinct helper-inducer T cells. Hum Immunol 1991;31:67-75.

8 Cattoretti G, Chang CC, Cechova K, et al. BCL-6 protein is expressed in germinal-center B cells. Blood 1995;86:45-53.

9 Nguyen PL, Ferry JA, Harris NL. Progressive transformation of germinal centers and nodular lymphocyte predominance Hodgkin's disease: a comparative immunohistochemical study. Am J Surg Pathol 1999;23:27-33.

10 Poppema S, Kaiserling E, Lennert K. Hodgkin's disease with lymphocytic predominance, nodular type (nodular paragranuloma) and progressively transformed germinal centres-a cytohistological study. Histopathology 1979;3:295-308.

11 Hansmann ML, Fellbaum C, Hui PK, et al. Progressive transformation of germinal centers with and without association to Hodgkin's disease. Am J Clin Pathol 1990;93:219-26.

12 Ho IC, Glimcher LH. Transcription: tantalizing times for T cells. Cell 2002;109(Suppl):S109-20.

13 Dorfman DM, Hwang ES, Shahsafaei A, et al. T-bet, a T-cell-associated transcription factor, is expressed in a subset of B-cell lymphoproliferative disorders. Am J Clin Pathol 2004; 122:292-7.

14 Atayar C, Poppema S, Blokzijl T, et al. Expression of the T-Cell transcription factors, GATA-3 and T-bet, in the neoplastic cells of Hodgkin lymphomas. Am J Pathol 2005; 166:127-34.

15 Dorfman DM, Hwang ES, Shahsafaei A, et al. T-bet, a T cell-associated transcription factor, is expressed in Hodgkin's lymphoma. Hum Pathol 2005;36:10-5.

16 Rudiger T, Gascoyne RD, Jaffe ES, et al. Workshop on the relationship between nodular lymphocyte predominant Hodgkin's lymphoma and T cell/histiocyte-rich B cell lymphoma. Ann Oncol 2002;13(Suppl 1):44-51.

17 Szabo SJ, Kim ST, Costa GL, et al. A novel transcription factor, T-bet, directs Th1 lineage commitment. Cell 2000;100:655-69.

18 Kaplan MH, Schindler U, Smiley ST, et al. Stat6 is required for mediating responses to IL-4 and for development of Th2 cells. Immunity 1996;4:313-9.

19 Zhang DH, Cohn L, Ray P, et al. Transcription factor GATA-3 is differentially expressed in murine Th1 and Th2 cells and controls Th2-specific expression of the interleukin-5 gene. J Biol Chem 1997;272:21597-603.

$20 \mathrm{Kim}$ JI, Ho IC, Grusby MJ, et al. The transcription factor c-Maf controls the production of interleukin-4 but not other Th2 cytokines. Immunity 1999;10:745-51.

21 Hurt EM, Wiestner A, Rosenwald A, et al. Overexpression of c-maf is a frequent oncogenic event in multiple myeloma that promotes proliferation and pathological interactions with bone marrow stroma. Cancer Cell 2004;5:191-9.

22 Durali D, de Goer de Herve MG, Giron-Michel J, et al. In human B cells, IL-12 triggers a cascade of molecular events similar to Th1 commitment. Blood 2003;102:4084-9.

23 Poppema S, van den Berg A. Interaction between host T cells and Reed-Sternberg cells in Hodgkin lymphomas. Semin Cancer Biol 2000; 10:345-50.

24 Afkarian M, Sedy JR, Yang J, et al. T-bet is a STAT1-induced regulator of IL-12R expression in naive CD4+ T cells. Nat Immunol 2002;3:549-57.

25 Stock $P$, Akbari $O$, Berry $G$, et al. Induction of T helper type 1-like regulatory cells that express Foxp3 and protect against airway hyper-reactivity. Nat Immunol 2004;5:1149-56.

26 Cousins DJ, Lee TH, Staynov DZ. Cytokine coexpression during human Th1/Th2 cell differentiation: direct evidence for coordinated expression of Th2 cytokines. $\mathrm{J}$ Immunol 2002;169:2498-506.

27 Chan WC. Cellular origin of nodular lymphocyte-predominant Hodgkin's lymphoma: immunophenotypic and molecular studies. Semin Hematol 1999;36:242-52

28 Carbone A, Gloghini A, Aldinucci D, et al. Expression pattern of MUM1/IRF4 in the spectrum of pathology of Hodgkin's disease. Br J Haematol 2002;117:366-72.

29 Rengarajan J, Mowen KA, McBride KD, et al. Interferon regulatory factor 4 (IRF4) interacts with NFATc2 to modulate interleukin 4 gene expression. J Exp Med 2002; 195:1003-12.

30 Kataoka K, Nishizawa M, Kawai S. Structure-function analysis of the maf oncogene product, a member of the b-Zip protein family. J Virol 1993;67:2133-41.

31 Chesi M, Bergsagel PL, Shonukan OO, et al. Frequent dysregulation of the c-maf proto-oncogene at $16 \mathrm{q} 23$ by translocation to an $\mathrm{lg}$ locus in multiple myeloma. Blood 1998;91:4457-63.

32 Delabie J, Vandenberghe E, Kennes C, et al. Histiocyte-rich B-cell lymphoma. A distinct clinicopathologic entity possibly related to lymphocyte predominant Hodgkin's disease, paragranuloma subtype. Am J Surg Pathol 1992;16:37-48.

33 Marafioti T, Mancini C, Ascani S, et al. Leukocyte-specific phosphoprotein-1 and PU. 1: two useful markers for distinguishing T-cell-rich B-cell lymphoma from lymphocyte-predominant Hodgkin's disease, Haematologica 2004;89:957-64. 